\title{
Apoplejía hipofisaria en posoperatorio inmediato de cirugía cardiaca: a propósito de un caso
}

\author{
Postoperative pituitary apoplexy: \\ case report after a cardiac surgery
}

Alfonso Hernández', David Velasco

\begin{abstract}
Pituitary apoplexy is caused by ischemia or hemorrhage and is considered a medical emergency. A percentage of undiagnosed pituitary adenomas may be diagnosed in the postoperative period of cardiac surgery. The clinical picture is a consequence of the increase in pressure which causes compression on cerebral structures (optic chiasm, cranial nerves) and hormonal deficit. We present a clinical case of a 69-year-old Caucasian male, who debuted with a clinical syndrome of the left cavernous sinus in the immediate postoperative period of cardiac surgery. Clinical and imaging studies (MRI) confirmed the diagnosis of pituitary apoplexy. The condition was solved satisfactorily with replacement therapy. She underwent urgery sixth days later due to persistence of visual symptoms.
\end{abstract}

\section{RESUMEN}

La apoplejía hipofisaria se produce por un infarto o una hemorragia en dicha glándula y se considera una emergencia médica. Un porcentaje de adenomas hipofisarios no diagnosticados debutan clínicamente así, pudiéndose producir en el postoperatorio de cirugía cardiaca. El cuadro clínico es consecuencia del aumento de presión y volumen en el interior de la silla turca, lo que origina compresión de las estructuras cerebrales colindantes (quiasma óptico, silla turca, nervios craneales, etc.) y déficit hormonal. Presentamos un caso clínico de un varón de raza caucásica de 69 años, el cual debuta con un síndrome clínico de

\section{Key words:}

Pittuitary apoplexy, cardiac surgery

\section{Palabras clave:}

Apoplejía hipofisaria, cirugía cardiaca

Servicio de Anestesiología y Reanimación en el Hospital Clínico Universitario de Valladolid. España.

Fecha de recepción: 27 de febrero de 2018

Fecha de aceptación: 23 de abril de 2018

ORDCID

https://orcid.org/0000-0001-7301-2131

Correspondencia:

Alfonso Hernández

Teléfono +34655483323

Email: alfonso-1989@hotmail.es 
Apoplejía hipofisaria en posoperatorio inmediato de cirugía cardiaca: a propósito de un caso - A. Hernández et al.

seno cavernoso izquierdo en el posoperatorio inmediato de una cirugía cardiaca. La clínica y los estudios de imagen (RMN) confirmaron el diagnóstico de apoplejía hipofisaria. El cuadro se resolvió satisfactoriamente con tratamiento esteroideo sustitutivo y cirugía al sexto día del inicio de los síntomas por persistencia de los síntomas visuales.

\section{Introducción}

E n 1898 Bailey fue el primero en describir una hemorragia cerebral asociada a un macroadenoma hipofisario, siendo Brougham en 1950 el autor que definió el término "apoplejía hipofisaria $(\mathrm{AH})$ " $[1],[2]$. Es una entidad infrecuente, con una prevalencia de 6 casos por 100.000 habitantes, más frecuente en pacientes mayores de 50 - 60 años y varones (2:1) [2],[3]. La AH es una situación clínica de urgencia médica, debida a la aparición aguda de una hemorragia y/o infarto en la hipófisis y que puede provocar compresión de estructuras cerebrales importantes tales como quiasma óptico, varios pares craneales, silla turca, seno cavernoso, etc. poniendo en compromiso vital al paciente[2],[4],[5]. Está descrito que hasta el $80 \%$ de pacientes afectos de un adenoma hipofisario debutan clínicamente con $\mathrm{AH}$. Otras veces la $\mathrm{AH}$ puede ocurrir espontáneamente, o ser secundaria a otras situaciones tales como fármacos (anticoagulantes, estrógenos, agonistas dopaminérgicos), diabetes mellitus, hipertensión arterial (HTA), embarazo, traumatismos craneoencefálicos, poscirugía, radioterapia[2],[4]-[6].

Las manifestaciones clínicas de la AH van a depender de la gravedad del insulto provocado a la glándula; algunas de ellas son:

- Cefalea, referida más frecuentemente con carácter retroorbitario[2],[4],[7].

- Defectos visuales, producidos normalmente por compresión nerviosa y que incluyen disminución de la agudeza visual, visión borrosa, fotofobia, oftalmoplejia, alteraciones campimétricas (siendo la más frecuente la hemianopsia bitemporal) $[2],[4],[5],[7]$.

- Otras manifestaciones son hipopituitarismo (más frecuente, hipocortisolemia), diabetes insípida, fiebre, náuseas, vómitos, etc.[2],[5],[7].

El diagnóstico se basa en la combinación de un cuadro clínico compatible con $\mathrm{AH}$ y la realización de una prueba de imagen siendo la resonancia magnética nuclear (RMN) de elección[2]-[6]. Debe realizar- se diagnóstico diferencial con otras entidades como hemorragia subaracnoidea, meningitis, infarto del tronco encefálico o trombosis del seno cavernoso[2],[4],[5].

Dado que la mayoría de los pacientes con $\mathrm{AH}$ presentan déficit de hormona corticotropa, algunos autores recomiendan el tratamiento con glucocorticoides (hidrocortisona) lo más precozmente posible[2],[5],[7],[8]. El tratamiento quirúrgico se reserva a los pacientes con alteración del nivel de conciencia y/o trastornos visuales graves de aparición brusca y carácter progresivo, y deberá realizarse preferentemente en los 7 primeros días desde su aparición. La técnica quirúrgica de elección debería ser la resección transesfenoidal[2],[9],[10].

\section{Caso clínico}

Presentamos el caso de un paciente varón de 69 años de edad $(76 \mathrm{~kg}, 171 \mathrm{~cm}$ ) con antecedentes personales de hipertensión arterial (HTA) y en tratamiento con ácido acetilsalićlico (AAS), bisoprolol, captopril y furosemida que ingresa en el servicio de cirugía cardiaca con el diagnóstico de "insuficiencia mitral severa y enfermedad coronaria de 1 vaso" para someterse a cirugía de sustitución valvular mitral más bypass aortocoronario. Clínicamente el paciente presentaba disnea de unos 5 meses de evolución en clase funcional II-III de la NYHA. La exploración física no mostraba hallazgos de interés salvo soplo en foco mitral. El ECG preoperatorio mostraba ritmo sinusal a $65 \mathrm{lpm}$ con extrasistolia ventricular ocasional. Radiografía de tórax con cardiomegalia a expensas de cavidades izquierdas. El ecocardiograma transtorácico mostraba como hallazgos más relevantes "aurícula izquierda dilatada con función ventricular ligeramente disminuida. Válvula mitral engrosada con imagen de masa filamentosa densidad media en cara auricular con velo anterior móvil e insuficiencia severa". En el ecocardiograma transesofágico se evidenciaba "aurícula izquierda dilatada (47 mm), ventrículo izquierdo dilatado (FEVI 47\%) con función global ligeramente 
deprimida, sin claras alteraciones de la dinámica segmentaria. VD con función conservada. Válvula mitral con insuficiencia severa (ORE 0,41, VC 0,8 índice área $52 \%$, reverso en vena pulmonar). Válvula aórtica trivalva fibrosada. IT trivial con PSAP $47 \mathrm{mmHg}$. VCl no dilatada con colapso inspiratorio". El cateterismo cardíaco mostraba enfermedad severa de DA media con estenosis del 75\%. En la analítica: $\mathrm{Hb}: 41,50 \mathrm{~g} / \mathrm{dl}$, $\mathrm{Ht}: 15,4 \%$, leucocitos: $7.100 / \mathrm{mm}^{3}$, plaquetas: $180 \mathrm{x}$ 103/dl, glucosa: $115 \mathrm{mg} / \mathrm{dl}, \mathrm{Cr}: 1,16 \mathrm{mg} / \mathrm{dl}, \mathrm{Na}: 138$ $\mathrm{mEq} / \mathrm{L}, \mathrm{K}: 3,7 \mathrm{mEq} / \mathrm{L}, \mathrm{Cl} 99 \mathrm{mEq} / \mathrm{L}$, urea: $52 \mathrm{mg} / \mathrm{dl}$, proteínas totales: $7 \mathrm{~g} / \mathrm{dl}$.

La intervención se realizó bajo anestesia general y circulación extracorpórea (CEC) efectuándose sustitución valvular mitral por prótesis mecánica tipo ATS (29 $\mathrm{mm}$ ) en posición intraanular más derivación aorto- coronaria con injerto de arteria mamaria interna izquierda a arteria descendente anterior. Intraoperatoriamente se objetivó degeneración con engrosamiento de velos en la válvula mitral degenerada con velos engrosados imposibilitando su reparación. La monitorización intra CEC se resume en la tabla siguiente:

$\begin{array}{lll}\text { Peso: } 76 & \text { Talla: } 171 & \text { S.C. 1,88 } \\ \text { T CEC 93 } & \text { T० Isquemia 68 } & \text { T Parada 0 } \\ \text { Hipotermia: } & \text { Desfibrilación: } & \text { Balance final: } \\ 35,50 & \text { Espontánea } & 2.240\end{array}$

Al finalizar la intervención se trasladó al paciente a la Unidad de Reanimación Cardiaca, intubado en ventilación mecánica, bajo efecto residual de anestesia general y con perfusión de milrinona y noradrenalina. Al ingreso el paciente es conectado a ventilación mecánica y se procede a monitorización con arterial radial más catéter de aurícula derecha más catéter de arteria pulmonar tipo Swan-Ganz para monitorización continua del gasto cardiaco.

Hemodinámicamente presentó el siguiente perfil:

$\begin{array}{lc}\text { Parámetro } & \text { Valor } \\ \text { Índice cardiaco } & 2,5 \\ \text { Presión venosa central } & 7 \\ \text { Presión capilar pulmonar } & 12 \\ \text { Presión arteria pulmonar media } & 18 \\ \text { Presión arterial media } & 75 \\ \text { Frecuencia cardiaca } & 90 \\ \text { Ritmo cardiaco } & \text { Sinusal }\end{array}$

Se procede a la retirada gradual del soporte inotrópico y vasopresor con buena tolerancia, pudiéndose extubar al paciente a las 4 horas. Durante la estancia en la Unidad presenta episodios de fibrilación auricular (FA) paroxística tratados con amiodarona.

La misma tarde del día del ingreso el paciente refiere un cuadro clínico consistente en cefalea hemicraneal izquierda con aparición de ptosis del ojo izquierdo (OI) que va aumentando de intensidad progresivamente. Es valorado por los Servicios de Oftalmología y Neurología, evidenciándose "fondo de OI normal acompañado de ptosis más oftalmoplejia del Ol salvo paresia incompleta a nivel del músculo recto externo y midriasis arreactiva con reflejo corneal preservado. Agudeza visual 0,2 en OI ". El resto de la exploración neurológica, ojo derecho incluido, resulta ser normal.

Se indica con carácter urgente la realización de tomografía computarizada (TC) cerebral con contraste i.v. (que no evidenció signos de lesiones isquémicas territoriales ni hemorrágicas agudas intra ni extracraneales). No se observan signos directos ni indirectos de lesiones ocupantes de espacio) y de Angio- TC de troncos supraaórticos (TSAo) y Polígono de Willis (informado como "arterias carótidas comunes, internas y externas permeables; principales ramas del Polígono de Willis permeables").

Con estos datos, se establece el diagnóstico de "Síndrome de seno cavernoso izquierdo a estudio", se indica Resonancia Magnética Nuclear (RMNu) cuando se hayan retirado los cables de marcapasos epicárdicos temporales y se pauta tratamiento con Prednisona oral a dosis de $1 \mathrm{mg} \times \mathrm{kg} \mathrm{c} / 24 \mathrm{~h}$ ante la posibilidad de que se trate de un cuadro de origen inflamatorio.

El paciente es dado de alta a planta de hospitalización desde la Unidad de Reanimación Cardiaca a las $48 \mathrm{~h}$ del ingreso. En planta la exploración física no evidenció sintomatología compatible referida con hiperproducción hormonal (estigmas Cushingoides, galactorrea, cambios de aspecto físico o peso atribuibles) persistiendo en el OI la ptosis palpebral y la midriasis. La cefalea se controló con paracetamol $1 \mathrm{~g} / 8 \mathrm{~h}$. La analítica con valores hormonales fue la siguiente (se indican los valores más relevantes): 


\section{PARÁMETROS}

$\begin{array}{ll}\text { CREATININA } & 0,9 \\ \text { GLUCOSA } & 72 \\ \text { SODIO } & 142,65 \\ \text { POTASIO } & \star 2,9 \\ \text { CLORUROS } & 103,00 \\ \text { HEMOGLOBINA } & \star 8,7 \\ \text { V. HEMATOCRITO } & \star 25,1 \\ \text { LEUCOCITOS } & 7,95 \\ \text { LINFOCITOS } & \star 16,0 \\ \text { NEUTRÓFILOS } & \star 77,5 \\ \text { T-3 Total } & \star 0,30 \\ \text { T-3 Libre } & * 1,16 \\ \text { T-4 Total } & * 4,66 \\ \text { T-4 Libre } & \star 0,87 \\ \text { TSH } & 0,93 \\ \text { PROLACTINA Basal } & 4,39 \\ \text { TESTOSTERONA TOTAL } & \star 32,73 \\ \text { PROLACTINA Basal } & 4,89 \\ \text { TESTOSTERONA TOTAL } & \star 32,78\end{array}$

\section{UNIDADES VAL.}

$\mathrm{mg} / \mathrm{dl}$
$\mathrm{mg} / \mathrm{dL}$
$\mathrm{mEqT}$
$\mathrm{mEq} / \mathrm{l}$
$\mathrm{mE} / \mathrm{l}$
$\mathrm{g} / \mathrm{dl}$
$\%$
$\mathrm{x} 10^{\wedge} 3 / \mu \mathrm{l}$
$\%$
$\%$
$\mathrm{ng} / \mathrm{ml}$
$\mathrm{pg} / \mathrm{ml}$
$\mu \mathrm{g} / \mathrm{dl}$
$\mathrm{ng} / \mathrm{dl}$
$\mu l \mathrm{U} / \mathrm{ml}$
$\mathrm{ng} / \mathrm{ml}$
$\mathrm{ng} / \mathrm{dL}$
$\mathrm{ng} / \mathrm{ml}$
$\mathrm{ng} / \mathrm{dL}$

\section{REFERENCIA}

$[0,6-1,4]$

$[60-110]$

$[135,00-153,00]$

$[3,5-5,3]$

$[90,00-110,00]$

$[12,0-18,0]$

$[38,0-52,0]$

$[4,00-10.00]$

$[20,0-51,0]$

$[42,0-76,0]$

$[0,83-2,00]$

$[2,04-4,40]$

$[5,10-14,10]$

$[0,93-1,71]$

$[0,27-4,20]$

$[4,04-15,20]$

$[193,00-740,00]$

$[4,04-15,20]$

$[193,00-740,00]$

Se han cambiado los valores de la prueba y los de referencia a $\mathrm{ng} / \mathrm{dL}$ (anteriormente eran expresados en $\mathrm{ng} / \mathrm{mL}$, el factor de conversión aplicado es ng/mL x 100 = ng/dL). desde 16/10/2014

$\begin{array}{ll}\text { DHEA-S } & * 16,61 \\ \text { CORTISOL } 9 \text { am } & * 2,20 \\ \text { ACTH } 9 \text { am } & 15,28 \\ \text { HGH Basal } & 0,24 \\ \text { SOMATOMEDINA-C(IGF-1) } & 95,10\end{array}$

VALORES DE REFERENCIA

0 - 4 años: $47-231 \mathrm{ng} / \mathrm{mL}$

4 - 10 años: 50 - 315

10 - 12 años: $95-460$

12 - 15 años: 95 - 512

15 - 17 años: $57-426$

Adultos: 55 - 360

Se decide mantener durante el ingreso tratamiento corticoideo por riesgo de insuficiencia corticotropa (ACTH).

Se realiza RMNU con y sin contraste intravenoso que se informa como "(..) Gran masa localizada en silla turca (..) con áreas de mayor hiperintensidad en ambas secuencias, en probable relación con focos de sangrado. (..) hallazgos sugestivos como primera posibilidad de macroadenoma hipofisario. (..) En la secuencia eco de gradiente es visible un pequeño foco hipointenso en el seno de la lesión descrita que probablemente corresponda a foco de sangrado".

$\begin{array}{ll}\mu \mathrm{g} / \mathrm{dl} & {[33,60-249,00]} \\ \mu \mathrm{g} / \mathrm{dl} & {[6,20-18,00]} \\ \mathrm{pg} / \mathrm{ml} & {[7,20-63,30]} \\ \mathrm{ng} / \mathrm{ml} & {[0,00-5,00]} \\ \mathrm{ng} / \mathrm{mL} & \end{array}$

$[33,60-249,00]$

$[7,20-63,30]$

$\mathrm{ng} / \mathrm{mL}$
Con estos hallazgos clínico -radiológicos se indica pase de Servicio a Neurocirugía (NCR) para tratamiento quirúrgico del macroadenoma hipofisario. Señalar que se cambia el tratamiento anticoagulante oral con dicumarínicos del paciente por heparina en infusión intravenosa.

Como incidencias durante el postoperatorio inmediato en planta de hospitalización, señalar que se realizó un ecocardiograma transtorácico (ETT) de control que evidenció "dilatación severa de aurícula izquierda. Ventrículo izquierdo dilatado con disfunción ventricular severa por hipocinesia difusa (FE 33\%). 


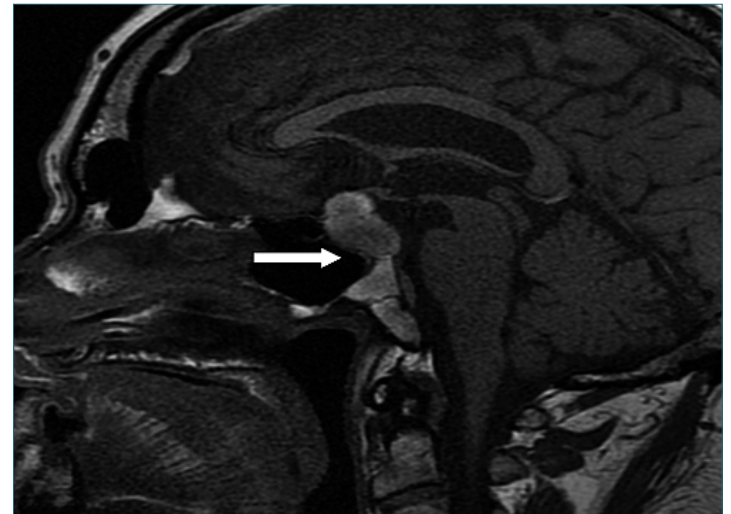

Figura 1. Imagen de RMNu sugestiva de adenoma hipofisario.

Prótesis mitral de gradientes adecuados sin que se observe insuficiencia por ecocardiograma transtorácico (..). Ventrículo derecho dilatado con función longitudinal conservada (..)" y que el paciente sufrió un episodio de fibrilación auricular (FA) postoperatoria que revierte a ritmo sinusal (RS) tras cardioversión eléctrica sincronizada a $200 \mathrm{~J}$.

A los 6 días del alta de la Unidad de Reanimación Cardiaca se procede a la intervención quirúrgica del macroadenoma hipofisario por parte de los Servicios de Anestesiología, Otorrinolaringología y Neurocirugía, realizándose bajo anestesia general "Abordaje endoscópico endonasal transesfenoidal y exéresis de la lesión".

La monitorización anestésica consistió en $\mathrm{SpO2}$, TA incruenta y cruenta, vía venosa central yugular interna derecha (YID), capnografía, ECG DII y V5, sondaje urinario, monitor de profundidad anestésica SEDLINE y manta térmica a $36^{\circ} \mathrm{C}$.

Como fármacos se administraron en la premedicación midazolam $2 \mathrm{mg}$, fentanilo $50 \mathrm{mcg}$; dexametasona 4 mg como antiemético ( el paciente mantenía su pauta de $1 \mathrm{mg} \mathrm{x} \mathrm{kg} \mathrm{c/24} \mathrm{h}$ de prednisona perioperatoria). La inducción se realizó con propofol $100 \mathrm{mg}$ y rocuronio $75 \mathrm{mg}$ y el mantenimiento con perfusión de propofol (4 - $6 \mathrm{mg} / \mathrm{kg} \times \mathrm{h}$ ), remifentanilo en perfusión a 0,05- 0,2 $\mathrm{mc} / \mathrm{kg} \times$ min y rocuronio en perfusión continua a $0,5 \mathrm{mg} / \mathrm{kg} \times \mathrm{h}$. Como medicación analgésica de rescate se pautó media hora antes del fin de la intervención paracetamol $1 \mathrm{~g}$, tramadol $100 \mathrm{mg}$ y cloruro mórfico $3 \mathrm{mg}$. Al final de la intervención, que duró aproximadamente $4 \mathrm{~h} \mathrm{30}$, se revirtió el bloqueo neuromuscular con 300 mg de Sugammadex y se extubó al paciente en quirófano sin complicaciones. Se

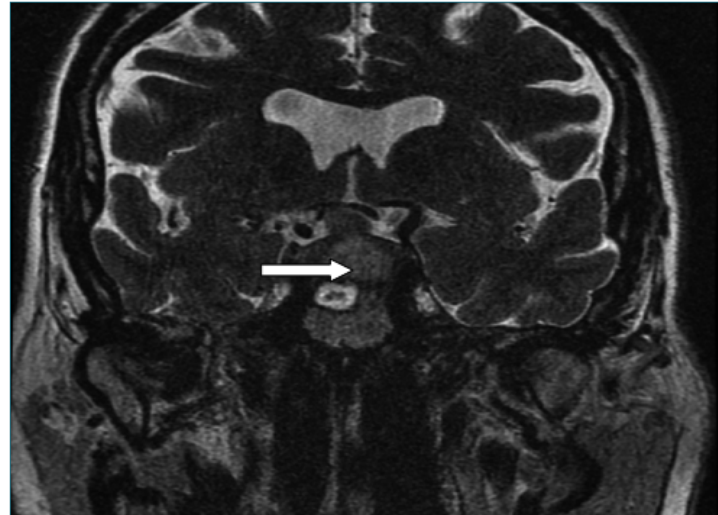

Figura 2. Corte coronal de la RMNu que evidencia foco hipofisario de sangrado.

administraron en total $500 \mathrm{ml} \mathrm{SSF}$ más el volumen de las perfusiones de fármacos. La diuresis total fue de $260 \mathrm{ml}$ sin diuréticos.

Reseñar que debido a los antecedentes cardiológicos del paciente no se emplearon lentinas empapadas en anestésico local ni en vasoconstrictor ni se realizó ningún tipo de infiltración a nivel de la mucosa nasal con estos fármacos por parte de la cirujana ORL. EI paciente presentó durante toda la intervención bradicardia sinusal entre 50 - 60 lpm que no respondió a la administración de atropina pero que por otra parte no se acompañó de ninguna otra alteración hemodinámica. Sí se observó tendencia a la HTA que se trató con la titulación cuidadosa de la perfusión de RMF sin precisar la administración de medicación cardiovascular.

Cabe destacar que en la gasometría arterial (GAR) postinducción se observó una hipopotasemia que se manejó administrando por vía central 50 meq de $\mathrm{KCl}$. Dicha hipopotasemia ya aparecía en la analítica preoperatoria y se corrigió en las $48 \mathrm{~h}$ de estancia en la Unidad de Reanimación General, donde fue trasladado el paciente para manejo del postoperatorio inmediato. Allí permaneció hemodinámica y ventilatoriamente estable, con una diuresis de 2.480 $\mathrm{ml}$ sin precisar diuréticos y pudiéndose dar de alta a las 48 horas a planta de hospitalización. Durante la estancia en planta el paciente presentó sangrado nasal tras retirada de taponamientos nasales, por lo que se ajustó tratamiento anticoagulante y se decide nuevo taponamiento nasal (durante 72 horas). Durante el ingreso es valorado, seguido y tratado por los servicios de endocrinología, cirugía cardiaca y hematología. 


\section{Discusión}

La hipófisis es una glándula situada en la silla turca del hueso esfenoides, que regula diferentes funciones hormonales del organismo[2]. Anatómicamente se relaciona con el quiasma óptico, el hipotálamo, las arterias carótidas internas, los senos cavernosos y los pares craneales III, IV, V y VI[2],[7]. Un 0,6 - 9\% de pacientes portadores de adenoma hipofisario desarrollarán $\mathrm{AH}$, y hasta un $80 \%$ de estos debutarán clínicamente con una $\mathrm{AH}$, situación que aconteció en el paciente que nos ocupa. La expresión clínica de la $\mathrm{AH}$ va a derivar del déficit hormonal producido y de la expansión del adenoma debido a la hemorragia o isquemia, lo que origina la compresión de las estructuras nerviosas circundantes[2],[4],[5]. En este caso el paciente debutó con cefalea y alteraciones de la motilidad y la agudeza visual aunque sin alteración del nivel de conciencia. El factor de riesgo identificado con más frecuencia es la HTA, existiendo también asociación con el perioperatorio de cirugías mayores (cardiaca sobre todo) y con la toma de medicación anticoagulante, circunstancias todas ellas que se daban en el paciente que nos ocupa. Se han postulado entre otros factores que la anticoagulación intraoperatoria, el flujo de bomba no pulsátil durante la circulación extracorpórea la lesión por reperfusión y las fluctuaciones en el estado hemodinámico podrían contribuir a la fisiopatología de la $\mathrm{AH}[12]$.

Ante la sospecha del cuadro, debe realizarse una $\mathrm{RMNu}$, prueba de imagen de elección que detectará con más precisión la imagen de AH que la TC. [2],[7]. También deben realizarse analíticas con niveles de hormonas de función hipofisaria, electrolitos y función renal y hepática. En este caso se decidió mantener tratamiento únicamente con prednisona sin aportar suplemento de hormona tiroidea habida cuenta de que el paciente presentaba disminución de agudeza visual con alteraciones en la motilidad ocular[5],[10]. El tratamiento quirúrgico consiste en la descompresión quirúrgica preferentemente por vía transesfenoidal, en pacientes con alteración del nivel de conciencia y/o alteraciones visuales persistentesgraves y realizada en los 7 primeros días tras la aparición de los síntomas[2],[5],[7],[8],[11]. Nuestro paciente fue intervenido a los 6 días desde la aparición del cuadro clínico y al presentar alteraciones visuales cumplía criterios para un tratamiento quirúrgico frente a un manejo más conservador, realizándose cirugía endoscópica nasosinusal por parte de los Servicios de Neurocirugía y Otorrinolaringología con una duración total aproximada incluido el tiempo anestésico de 4 horas 30 minutos y transcurriendo sin complicaciones.

\section{Conclusiones}

La $\mathrm{AH}$ en el postoperatorio inmediato de cirugía cardiaca es una entidad rara, pero potencialmente mortal. Clínicamente, se caracteriza por la aparición brusca de cefalea, defectos visuales y alteración en el nivel de conciencia[6]. Ante la sospecha se debe realizar una RMNu tan pronto como se pueda para el diagnóstico diferencial con otras entidades (meningitis, hemorragia subaracnoidea)[7]. El tratamiento inicial empírico con glucocorticoides puede salvar la vida al paciente[5]. Deberá considerarse el tratamiento quirúrgico en los pacientes con deterioro clínico, visual o del nivel de conciencia preferentemente en los 7 primeros días desde el inicio de los sínto$\operatorname{mas}[2],[7],[9],[10],[11]$.

\section{Referencias}

1. Oldfield EH, Merrill MJ. Apoplexy of pituitary adenomas: the perfect storm. J Neurosurg. 2015 Jun;122(6):1444-9.

2. Briet $C$, Salenave $S$, Bonneville JF, Laws ER, Chanson P. Pituitary Apoplexy. Endocr Rev. 2015 Dec;36(6):622-45.

3. Billeci D, Marton E, Giordan E. Post-traumatic pituitary apoplexy: case presentation and review of literature. Interdiscip Neurosurg. 2017;7:4-8.

4. Singh TD, Valizadeh $N$, Meyer $F B$, Atkinson JL, Erickson D, Rabinstein AA. Management and outcomes of pituitary apoplexy. J Neurosurg. 2015 Jun;122(6):1450-7.

5. Baldeweg SE, Vanderpump $M$, Drake W, Reddy N, Markey A, Plant GT, et al.; Society for Endocrinology Clinical Committee. SOCIETY FOR ENDOCRINOLO-
GY ENDOCRINE EMERGENCY GUIDANCE: emergency management of pituitary apoplexy in adult patients. Endocr Connect. 2016 Sep;5(5):G12-5.

6. Francis F, Burger I, Poll EM, Reineke A, Strasburger CJ, Dohmen $\mathrm{G}$, et al. Can cardiac surgery cause hypopituitarism? Pituitary. 2012 Mar;15(1):30-6.

7. Nawar RN, AbdelMannan D, Selman WR, Arafah BM. Pituitary tumor apoplexy: a review. J 
Intensive Care Med. 2008 MarApr;23(2):75-90.

8. Chanson P, Lepeintre JF, Ducreux D. Management of pituitary apoplexy. Expert Opin Pharmacother. 2004 Jun;5(6):1287-98.

9. Kanter AS, Dumont AS, Asthagiri AR, Oskouian RJ, Jane JA Jr, Laws ER Jr. The transsphenoidal approach. A historical perspective. Neurosurg Focus. 2005 Apr;18(4):e6.

10. Rajasekaran S, Vanderpump M, Baldeweg S, Drake W, Reddy N, Lanyon $\mathrm{M}$, et al. UK guidelines for the management of pituitary apoplexy. Clin Endocrinol (Oxf).
2011 Jan;74(1):9-20.

11. Vicente $A$, Lecumberri $B$, Gálvez MÁ. Guía de práctica clínica para el diagnóstico y tratamiento de la apoplejía hipofisaria. Endocrinología y Nutrición [Internet]. Elsevier BV; 2013 Dec;60(10):582. e1-582.e12. 